\title{
Facteurs d'adoption de la haie vive améliorée au Mali
}

\author{
Virginie Levasseur ${ }^{2}$ \\ Alain Olivier ${ }^{1}$ \\ Steven Franzel $\left.\right|^{3}$ \\ ${ }^{1}$ Département de phytologie \\ Pavillon Paul-Comtois \\ Université Laval \\ G1K 7P4 Québec \\ Canada \\ <alain.olivier@fsaa.ulaval.ca> \\ 2 Université de Moncton \\ Faculté de foresterie \\ Campus d'Edmundston \\ E3V 2S8 Edmundston \\ Canada \\ <vlevass@umce.ca> \\ ${ }^{3}$ World Agroforestry Centre \\ United Nation Avenue \\ Gigiri PO Box 30677-00100 \\ Nairobi \\ Kenya \\ <s.franzel@cgiar.org>
}

\begin{abstract}
Résumé
Depuis quelques années, on assiste au Mali à une augmentation de la production marấchère et de la culture de manioc au cours de la saison sèche. Traditionnellement, ces cultures sont protégées des animaux d'élevage, qui divaguent librement à cette époque de l'année, par des clôtures faites de branches épineuses collectées sur divers arbres, les haies mortes. Pour diminuer la pression que ce prélèvement entraîne sur les arbres du terroir, certains proposent l'utilisation par les paysans de la haie vive améliorée, une clôture d'arbres vivants, généralement épineux, plantés à très faible espacement. Diverses contraintes limitent toutefois l'adoption de cette technique par les paysans. Une étude a donc été réalisée dans le Cercle de Ségou, afin de caractériser les Unités de production agricole (UPA) utilisant une haie vive améliorée et d'identifier les facteurs déterminant son adoption. Les UPA utilisant une haie vive améliorée possèdent généralement plus de main-d'œuvre, plus de matériel agricole et un cheptel animal plus important que les UPA non utilisatrices. Le fait que les UPA utilisatrices de la haie vive améliorée fassent partie des UPA les mieux nanties soulève des interrogations quant à son accessibilité aux paysans les plus démunis. Les résultats indiquent par ailleurs que l'utilisation de la technique est déterminée par des variables liées à l'information et à la formation des paysans.
\end{abstract}

Mots clés : adoption de l'innovation ; culture maraîchère ; haie ; main-d'œuvre ; Mali.

Thèmes : mécanisation et aménagement rural ; productions végétales ; systèmes agraires.

\section{Abstract}

\section{Factors affecting adoption of the improved living hedge in Mali}

Over the last few years, in Mali, vegetable and cassava production during the dry season have been increasing. Traditionally, these crops were protected from roaming livestock by dead fences made of thorny tree branches. In order to reduce the pressure on the woody component of the surrounding parklands, some experts promote the use of live fences made of multipurpose fast growing trees planted close to one another around the plot as an alternative to dead fences. However, various constraints impede the adoption of live fences by farmers. A study was therefore undertaken in the Segou area in order to characterize the Agricultural Production Units (APU) using a live fence and to identify the factors determining its adoption. The APU that use live fences have access to more labour force, more agricultural equipment and more livestock than non users. The fact that these factors are typical of the wealthiest APU raises concern about the overall accessibility of this agroforestry technique for the poorest farmers. Results also show that the use of live fences is determined by variables related to information and training of farmers.

Key words: hedges; innovation adoption; Mali; manpower; vegetable growing.

Subjects: farming systems; mechanization and country planning; vegetal productions. e Mali a vu sa population urbaine augmenter considérablement au cours des dernières décennies. Néanmoins, contrairement aux prévisions pessimistes de certains économistes, les agriculteurs ont été en mesure d'offrir aux citadins des produits vivriers en quantité suffisante et à des prix convenables 
(Pélissier, 2000). On a notamment observé un véritable essor des productions marầchères et de la culture de manioc en saison sèche. Or, les cultures de saison sèche sont menacées par le bétail, qui circule librement dans les champs durant cette saison, en vertu d'ententes immémoriales entre éleveurs et agriculteurs qui permettent aux animaux d'élevage de profiter des résidus de culture.

Toute production agricole en saison sèche doit donc être protégée des animaux d'élevage. Habituellement, les paysans érigent pour ce faire des haies mortes, c'est-à-dire des clôtures faites de branches épineuses collectées sur divers arbres (figure 1) ou de résidus de culture. Ce type de protection est d'une efficacité restreinte, puisque la haie morte subit l'attaque des termites et doit être reconstruite chaque année.

Certains paysans utilisent également des haies vives, le plus souvent constituées d'Euphorbia balsamifera ou de Jatropha curcas (figure 2). Depuis quelques années, l'ICRAF (World Agroforestry Centre) et l'IER (Institut d'économie rurale) proposent une haie vive dite "améliorée ", qui est composée d'arbres à usages multiples, généralement épineux, qui sont densément plantés autour de la parcelle à protéger. Trois à cinq ans après son installation, la haie vive améliorée offre une protection durable aux cultures, tout en favorisant la conservation de la ressource arborée. On suppose également qu'une meilleure protection des cultures devrait permettre l'obtention de meilleurs rendements et, dès lors, un surplus de production pouvant être vendu sur le marché (Ayuk, 1997). À la suite d'enquêtes réalisées auprès des agriculteurs pour connaître leurs préférences, les espèces d'arbres proposées par l'ICRAF pour confectionner la haie vive améliorée sont: Ziziphus mauritiana (jujubier), Acacia nilotica, Acacia senegal, Lawsonia inermis (henné) et Baubinia rufescens (figure 3).

Une étude exploratoire, menée auprès des 20 premiers utilisateurs de la haie vive améliorée, a révélé que ceux-ci se déclarent satisfaits de la protection qu'elle offre et des nombreux produits qu'elle procure (Levasseur et al., 2004). Cependant, plusieurs contraintes limitent son adoption. Parmi celles-ci, on retrouve divers aspects liés aux modes d'accès à la terre et à la signification des arbres plantés en bordure de parcelles (Levasseur et al., 2008).
Conséquemment, la présente étude a été entreprise afin d'identifier, d'une part, les principales caractéristiques des Unités de production agricole (UPA) qui ont réussi à surmonter ces contraintes et utilisent la haie vive améliorée et, d'autre part, les facteurs qui déterminent l'adoption de cette nouvelle technique. Les résultats

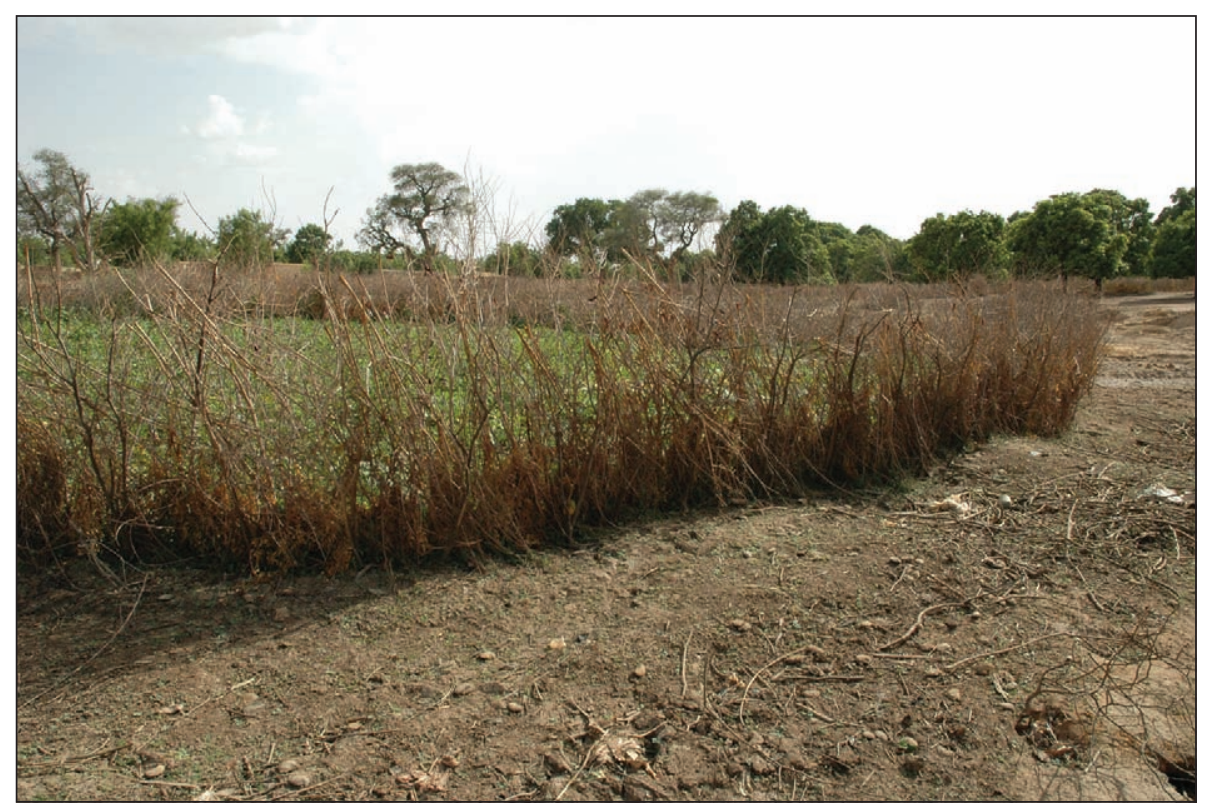

Figure 1. Haie morte constituée de branchages pour la protection de parcelles maraîchères.

Figure 1. Dead fence composed of branches for the protection of vegetable plots.. Photo : Isabelle Winter.

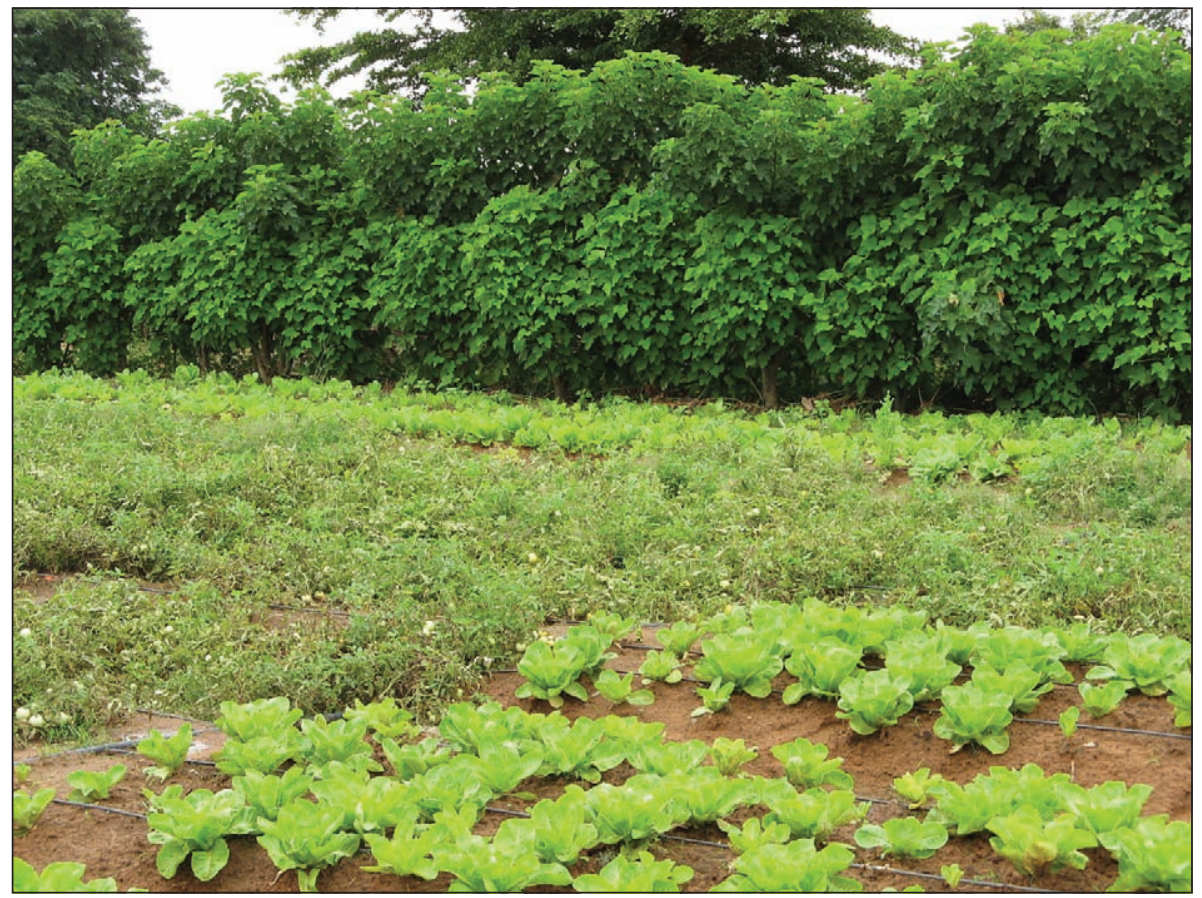

Figure 2. Jardin potager protégé par une haie vive traditionnelle composée de Jatropha curcas.

Figure 2. Market garden protected by a traditional live fence made of Jatropha curcas. Photo : Virginie Levasseur. 
de cette étude devraient permettre de mieux comprendre le processus d'adoption de la haie vive améliorée et, par conséquent, de mieux cibler les interventions en vue de sa diffusion.

\section{Méthodologie}

\section{Zone d'étude}

Depuis 1996, l'ICRAF fait la promotion de la haie vive améliorée dans le Cercle de Ségou. La collecte de données a donc été réalisée dans cette zone, de novembre 2000 à novembre 2001. Le Cercle de Ségou s'étend sur une superficie de $10844 \mathrm{~km}$ carrés. Sa population rurale était estimée à 201096 habitants en 1999. La zone est caractérisée par un climat de type soudano-sahélien. De 1994 à 1998, la pluviométrie annuelle moyenne y était de $586,2 \mathrm{~mm}$, répartis sur 44 jours pendant les mois de juillet, août et septembre. Le relief, plat, comprend de vastes plaines et quelques plateaux. Les sols situés le long du fleuve Niger sont de type hydromorphes, alors que les sols ferrugineux tropicaux dominent partout ailleurs Le petit mil (Pennisetum typhoides) et le

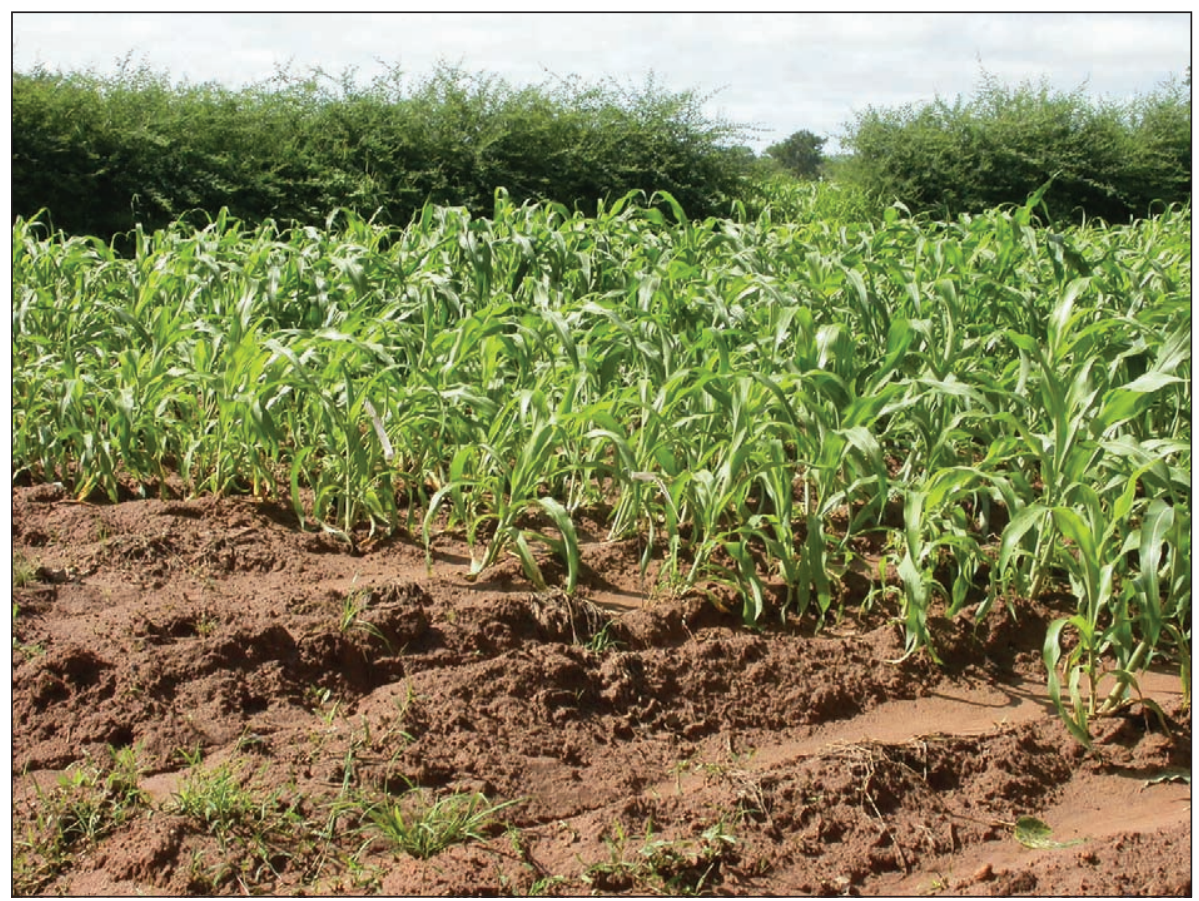

Figure 3. Champ de mil bordé par une haie vive améliorée constituée de Bauhinia rufescens.

Figure 3. Pearl millet field surrounded by an improved live fence made of Bauhinia rufescens. Photo : Virginie Levasseur. sorgho (Sorghum bicolor) sont les principales cultures produites dans la zone, suivies du riz (Oryza sativa), du niébé (Vigna unguiculata), du mais (Zea mays), du fonio (Digitaria exilis) et du voandzou (Voandzou subterranea).

\section{Collecte de données}

Les villages où l'étude a été réalisée ont été choisis parmi les villages ayant été ciblés par l'ICRAF pour la promotion de la haie vive améliorée. Trois critères qui nous paraissaient liés à l'utilisation de la technique ont été utilisés pour leur stratification, à savoir la pression démographique, la rareté de la ressource ligneuse et les productions agricoles, ce qui a permis de retenir huit villages présentant diverses combinaisons de ces critères: Bougounina, Dakala, Djigo, Dougoukouna, N'Tobougou, Sikila, Tesseribougou et Zogofina. Le travail d'enquête, dans chacun de ces villages, a été mené au niveau de l'UPA, ou chiké gwa, en langue bamana. Une UPA regroupe une partie ou l'ensemble des membres du groupe familial, vivant ou non sous le même toit, qui cultivent des champs en commun. L'échantillon comprenait 126 UPA, dont 51 utilisatrices de la technique, réparties au sein des huit villages sélectionnés pour l'étude. Afin de

les caractériser, ces UPA ont été soumises à un questionnaire portant sur différentes variables ayant un lien potentiel avec l'utilisation de la nouvelle technique : structure de la famille et sa composition, vie associative de ses membres, activités de production agricole au cours de la saison des pluies et de la saison sèche, matériel agricole disponible, utilisation des ressources ligneuses.

Finalement, à l'aide d'informateurs clés, nous avons procédé à un classement des UPA selon leur niveau de prospérité socio-économique (plus nanties, moyennement nanties et moins nanties) dans chacun des villages, en utilisant des critères de prospérité préalablement déterminés par les paysans eux-mêmes : possession d'une unité de labour, possession de boeufs d'élevage et atteinte de l'autosuffisance alimentaire.

\section{Analyse des résultats}

Les diverses variables quantitatives recueillies auprès de chaque UPA ont été mises en relation avec la présence ou l'absence de haies vives améliorées, au moyen d'un test de $t$ (pour les variables continues) ou de $\mathrm{X}^{2}$ (pour les variables catégoriques), au seuil de probabilité de $P=0,05$. Ces tests ont été réalisés à l'aide du logiciel SPSS (version 10.0 pour Windows). L'analyse a permis de caractériser les UPA utilisatrices de la haie vive améliorée et de les comparer à celles qui ne l'utilisent pas.

Les mêmes variables ont ensuite été analysées à l'aide d'un modèle logistique de régression linéaire (Hosmer et Lemeshow, 1989). À l'instar d'autres auteurs ayant eu recours à des modèles statistiques pour identifier les facteurs qui déterminent l'adoption d'une nouvelle technique (Gockowski et Ndoumbé, 2004 ; Sanginga et al., 2007), on a utilisé ce modèle logistique afin d'identifier quels sont les facteurs qui déterminent l'utilisation de la haie vive améliorée.

\section{Résultats et discussion}

\section{Portrait des UPA utilisant une haie vive améliorée}

Parmi les 22 caractéristiques des UPA qui ont été analysées, 17 ont présenté des différences significatives entre les UPA utilisatrices et non utilisatrices de la haie 
vive améliorée (tableau 1). Les variables ayant trait à la disponibilité de la maind'œuvre affichent des valeurs moyennes plus élevées chez les UPA utilisatrices que chez les UPA non utilisatrices. Par ailleurs, les UPA utilisatrices comptent en moyenne plus d'un ménage, ainsi que près de 10 membres supplémentaires, dont plus de 6 hommes et femmes actifs. Ces résultats montrent une relation entre la main-d'œuvre et l'utilisation de la haie vive améliorée. Cette technique est effectivement très exigeante en main-d'œuvre, notamment au début de la saison des pluies, quand les agriculteurs sont submergés par les travaux champêtres. Divers auteurs ont identifié le manque de main-d'œuvre comme une contrainte pour l'adoption de la haie vive améliorée (Sanogo, 2000). En revanche, Ayuk (1997) a rapporté que la disponibilité de la main-d'œuvre était négativement corrélée avec l'adoption de la haie vive améliorée au Burkina Faso. Selon lui, les petites UPA seraient plus enclines à installer une haie vive améliorée, afin de maximiser l'utilisation de la maind'œuvre. Dans notre étude, cependant, rien ne semble indiquer l'existence d'un tel phénomène.

L'analyse des données du tableau 1 indique par ailleurs que les UPA utilisant une haie vive améliorée possèdent plus de matériel agricole que les non utilisatrices. Elles disposent en moyenne de 0,5 multiculteur et 1,5 bouf de labour de plus. Elles possèdent également, en moyenne, presque un âne de plus. Ces résultats permettent de supposer qu'il est plus facile pour le chef d'UPA, dans un tel cas, de mobiliser une partie de la main-d'oeuvre et de l'équipement nécessaires à l'installation d'une haie vive, sans que les travaux qui doivent avoir lieu dans les champs collectifs en soient affectés.

Au plan du cheptel animal, on remarque que les UPA utilisatrices ont en moyenne
4 bœufs d'élevage de plus que les autres UPA. Elles ont également près de 4 ovins, plus de 3 caprins et près de 6 volailles de plus. Cela indique que les UPA utilisant une haie vive améliorée ont un meilleur revenu, les animaux constituant souvent l'épargne de l'UPA.

Les UPA utilisant une haie vive améliorée ont également une production sensiblement plus diversifiée en saison des pluies. Or, la diversification de la production agricole permet généralement de limiter les risques liés aux aléas climatiques, biologiques et économiques. Par ailleurs, bien qu'elles ne cultivent pas plus de cultures maraîchères et de manioc que les autres UPA, les UPA utilisatrices d'une haie vive améliorée commercialisent une plus grande proportion de leur production de manioc. Cela pourrait les inciter à entreprendre des démarches pour protéger efficacement leurs parcelles. Il convient cependant de préciser que les données recueillies n'indiquent pas si la parcelle

\section{Tableau 1. Valeur moyenne pour différentes caractéristiques d'Unités de production agricole (UPA) utilisant ou non une haie vive améliorée dans le Cercle de Ségou, Mali $(n=126)$.}

Table 1. Mean value for various characteristics of Agricultural Production Units (APU) using or not using improved live fences in the Segou area, Mali ( $n=126$ APU).

\begin{tabular}{|c|c|c|c|}
\hline & $\begin{array}{c}\text { UPA } \\
\text { utilisatrices } \\
(\mathrm{n}=51)\end{array}$ & $\begin{array}{c}\text { UPA non } \\
\text { utilisatrices } \\
(\mathbf{n}=75)\end{array}$ & $\begin{array}{c}\text { Test de } t \\
\text { ou } X^{2}\end{array}$ \\
\hline Nombre de ménages & $4,3(3,2)^{a}$ & $3,0(2,0)$ & $-2,752(0,007)^{\mathrm{b}}$ \\
\hline Nombre total de membres & $28,1(25,2)$ & $18,2(14,4)$ & $-2,766(0,007)$ \\
\hline Nombre d'hommes actifs & $9,3(8,3)$ & $6,2(4,9)$ & $-2,599(0,010)$ \\
\hline Nombre de femmes actives & $9,4(7,2)$ & $6,0(4,9)$ & $-3,070(0,003)$ \\
\hline Nombre de multiculteurs & $1,6(1,8)$ & $1,1(0,8)$ & $-2,065(0,043)$ \\
\hline Nombre de bœufs de labour & $4,0(4,0)$ & $2,5(2,5)$ & $-2,674(0,009)$ \\
\hline Nombre de charrettes & $2,4(2,8)$ & $1,8(1,5)$ & $-1,749(0,083)$ \\
\hline Nombre d'ânes & $2,9(3,3)$ & $1,9(1,6)$ & $-2,183(0,031)$ \\
\hline Nombre de cultures produites & $5,2(1,4)$ & $4,6(1,6)$ & $-2,580(0,011)$ \\
\hline Superficie totale en culture & $10,9(10,5)$ & $7,7(7,6)$ & $-1,855(0,067)$ \\
\hline Superficie en maraîchage & $0,2(0,5)$ & $0,2(0,3)$ & $-0,639(0,525)$ \\
\hline Pourcentage des produits maraîchers commercialisé & $47,0(41,5)$ & $45,6(40,5)$ & $-0,188(0,852)$ \\
\hline Superficie en manioc & $0,7(1,5)$ & $0,4(1,2)$ & $-0,921(0,359)$ \\
\hline Pourcentage du manioc commercialisé & $44,4(37,6)$ & $29,7(37,2)$ & $-2,160(0,033)$ \\
\hline Nombre de bœufs d'élevage & $5,6(11,3)$ & $1,6(3,9)$ & $-2,854(0,005)$ \\
\hline Nombre d'ovins & $7,1(6,9)$ & $3,2(3,4)$ & $-4,263(<0,001)$ \\
\hline Nombre de caprins & $7,2(7,7)$ & $3,6(4,5)$ & $-3,270(0,001)$ \\
\hline Nombre de volailles & $17,5(19,2)$ & $11,6(10,0)$ & $-2,252(0,026)$ \\
\hline Nombre de sources d'information & $2,8(0,8)$ & $2,4(1,0)$ & $-2,271(0,025)$ \\
\hline Pourcentage des UPA écoutant la radio & $90,2(4,2)$ & $70,7(5,2)$ & $6,877(0,009)$ \\
\hline Pourcentage des UPA ayant participé aux séances de I'ICRAF & $98,0(1,9)$ & $22,7(4,8)$ & $69,263(<0,001)$ \\
\hline Nombre d'associations dont I'UPA est membre & $3,6(1,2)$ & $2,3(1,3)$ & $-5,680(<0,001)$ \\
\hline
\end{tabular}

a Écart type ; pour toutes les analyses; degrés de liberté $=124 .{ }^{\mathrm{b}}$ La valeur de la probabilité $P$ associée au test de $\mathrm{t}$ (variables continues) ou au test de $X^{2}$ (variables catégoriques) est indiquée entre parenthèses. 
protégée par la haie vive améliorée est effectivement celle de manioc. En fait, dans bien des cas, la haie vive améliorée répondrait plus à un besoin de délimitation et d'appropriation des parcelles qu'à un simple besoin de protection des cultures (Levasseur et al., 2008).

Soulignons enfin que les UPA utilisant une haie vive améliorée consultent plus de sources d'informations, écoutent davantage la radio pour obtenir ces informations, sont membres d'un plus grand nombre d'associations liées à des opérations de développement rural et ont presque toutes participé aux séances d'information et de formation de l'ICRAF. L'information et la formation des membres de l'UPA semblent donc être des éléments importants dans le processus d'utilisation de cette nouvelle technique.

Plusieurs des caractéristiques que nous venons de mentionner, notamment la disponibilité de la main-d'oeuvre et de tance du cheptel animal, constituent, aux yeux mêmes des paysans, des critères représentatifs de leur niveau de prospérité. Il semble donc, à première vue, que l'équipement agricole ainsi que l'impor-

les UPA utilisatrices de la haie vive améliorée se trouvent parmi les mieux nanties. Pour vérifier cela, nous avons tenté de voir s'il existait des différences de classement socio-économique entre les UPA utilisatrices et non utilisatrices (tableau 2). La distribution des UPA au sein des différentes classes socio-économiques varie effectivement de façon significative entre ces deux groupes. Les UPA qui utilisent la haie vive améliorée se retrouvent pour la très grande majorité parmi les mieux ou moyennement nanties, alors que les UPA non utilisatrices sont réparties entre les différentes classes selon une courbe à distribution normale.

\section{Facteurs déterminant I'utilisation de la haie vive améliorée}

Selon le modèle de Hosmer et Lemeshow (1989) que nous avons retenu pour analyser nos données, deux facteurs, parmi les 22 variables soumises à l'analyse, déterminent l'utilisation de la haie vive améliorée (tableau 3). Le plus détermi-

\section{Tableau 2. Classement socio-économique des Unités de production agricole (UPA) en fonction de I'utilisation ou non d'une haie vive améliorée $(n=126)$ dans le Cercle de Ségou, Mali.}

Table 2. Socio-economic classification of Agricultural Production Units (APU) in relation to the use or not of improved live fences ( $n=126$ APU) in the Segou area, in Mali.

\begin{tabular}{lcc}
\hline Classe socio-économique & $\begin{array}{c}\text { UPA utilisant une haie } \\
\text { vive améliorée } \\
(\mathbf{\%})\end{array}$ & $\begin{array}{c}\text { UPA n'utilisant pas } \\
\text { de haie vive améliorée } \\
\text { (\%) }\end{array}$ \\
\hline UPA les plus nanties & 39,2 & 24,0 \\
UPA moyennement nanties & 52,9 & 52,0 \\
UPA les moins nanties & 7,8 & 24,0 \\
\hline
\end{tabular}

$X^{2}=6,874 ; P=0,032$

\section{Tableau 3. Facteurs déterminant l'utilisation de la haie vive améliorée dans le Cercle de Ségou, Mali, selon le modèle de régression logistique dichotomique.}

Table 3. Factors determining the use of improved live fences in the Segou area, Mali, according to the dichotomic logistic regression model.

\begin{tabular}{lc}
\hline Variable/modèle & $\mathbf{n = 1 2 6}$ \\
\hline Constante & $-5,6791(<0,0001)^{a}$ \\
Participation aux séances de I'ICRAF & $5,3251(<0,0001)$ \\
Écoute de la radio & $1,8643(0,0058)$
\end{tabular}

a La valeur de la probabilité $P$ est indiquée entre parenthèses. Bonne classification $=81,7 \%$. Spécificité $=77,3 \%$. Sensitivité $=88,2 \%$. nant est la participation des UPA aux séances d'informations et de formation de l'ICRAF. L'importance d'une telle participation semble aller de soi, même si cette participation n'était pas requise et n'entraînait pas nécessairement l'utilisation de la haie vive améliorée. Un tel résultat indique notamment que le processus d'adoption de la haie vive améliorée n'en serait qu'à ses débuts. Il souligne aussi l'importance de la vulgarisation dans le processus d'adoption de nouvelles techniques agricoles.

Le second facteur déterminant l'utilisation des haies vives améliorées est l'écoute de la radio afin d'obtenir des renseignements sur l'agriculture. Pourtant, au moment de l'enquête, l'ICRAF n'avait encore jamais mené de campagne d'information sur ses activités par le biais de la radio.

Les résultats de l'analyse logistique nous ramènent donc à la théorie de la diffusion des innovations de Rogers (2003), selon laquelle la communication de l'information est au centre de la diffusion. La participation des individus à des groupes de discussion et à des ateliers de formation, de même que l'échange d'informations avec d'autres membres de la communauté, sont des facteurs qui servent de catalyseurs à l'utilisation de nouvelles techniques agroforestières (Mercer, 2004 ; Sanginga et al., 2007).

\section{Conclusion}

Les résultats de la présente étude indiquent que les UPA utilisant une haie vive améliorée font partie des UPA les plus nanties des villages à l'étude. De nombreuses recherches ont démontré que les premiers utilisateurs d'une nouvelle technique - et en particulier d'une technique agroforestière - proviennent des couches les plus favorisées des sociétés rurales (Snelder et al., 2007), qui semblent plus enclines à l'innovation et à la prise de risque qu'elle comporte. Dans notre étude, les UPA utilisatrices de la haie vive améliorée bénéficient effectivement, pour subvenir à leurs besoins alimentaires et monétaires, de plus grands moyens de production que les UPA non utilisatrices. Elles disposent notamment de plus de main-d'oeuvre et d'équipement agricole, ce qui leur donne plus de souplesse dans l'organisation du travail, facteur qui a une influence déterminante lors de l'installation de la haie vive amé- 
liorée, qui doit se faire au même moment que le semis des céréales (Levasseur et al., 2008). Dès lors, ces UPA sont plus disposées à prendre le risque d'innover.

Le processus d'adoption de la haie vive améliorée est relativement récent dans le Cercle de Ségou. Les UPA qui en font l'utilisation ont donc encore, jusqu'à présent, des caractéristiques propres aux premiers utilisateurs d'une nouvelle technique. En plus de disposer de plus de moyens de production, ces UPA sont plus et mieux informées. Ainsi, les deux facteurs déterminant l'adoption de la haie vive améliorée, selon le modèle logistique utilisé, sont liés à l'information, un facteur souvent rapporté comme étant déterminant dans l'utilisation d'une nouvelle technique.

Il sera dorénavant intéressant d'observer quel sera le profil des UPA qui utiliseront la haie vive améliorée au cours des prochaines années. Assisterons-nous à une diffusion plus large de la technique, y compris auprès des paysans les plus démunis? L'introduction d'une nouvelle technique ne profite pas toujours à tous. La haie vive améliorée a d'abord été adoptée par les UPA les plus nanties. Si la tendance se maintient, elle pourrait représenter une source additionnelle de différenciation sociale et économique, à moins que les UPA les moins nanties puissent, elles aussi, profiter de ce type d'aménagement foncier.
Une plus grande démocratisation de la haie vive améliorée pourrait notamment passer par son installation dans des zones collectives, par exemple en bordure des chemins utilisés pour le passage des animaux d'élevage, ou autour des périmètres maraîchers collectifs qui sont le plus souvent sous la responsabilité des femmes. L'analyse des aspects fonciers liés à l'utilisation de la haie vive améliorée (Levasseur et al., 2008) souligne en effet la dimension collective d'une telle technique, un aspect qui ne devrait pas être négligé lors des activités qui, au Mali, visent à sa diffusion.

\section{Remerciements}

Les auteurs tiennent à remercier les paysans et paysannes qui ont participé à cette étude. Des remerciements particuliers doivent être adressés à Zoumana Sao pour son travail d'enquêteur et interprète, ainsi qu'à tout le personnel de l'ICRAF (World Agroforestry Centre) et de l'Institut d'économie rurale (IER) au Mali, pour son soutien. Cette étude a été rendue possible grâce à la contribution financière du Centre de recherches pour le développement international (CRDI).

\section{Références}

Ayuk ET. Adoption of agroforestry technology : the case of live hedges in the Central Plateau of Burkina Faso. Agr Syst 1997; 54: 189-206.

Gockowski J, Ndoumbé M. The adoption of intensive monocrop horticulture in southern Cameroun. Agr Econ 2004 ; 30 : 195-202.

Hosmer DW, Lemeshow S. Applied logistic regression. New York: John Wiley, 1989.

Levasseur V, Djimdé M, Olivier A. Live fences in Segou: an evaluation by their early users. Agroforest Syst $2004 ; 60$ : 131-6.

Levasseur V, Olivier A, Niang A. Aspects fonciers liés à I'utilisation de la haie vive améliorée. Bois et Forêts des Tropiques 2008; 297 : 55-64.

Mercer DE. Adoption of agroforestry innovations in the tropics: a review. Agroforest Syst $2004 ; 61: 311-28$

Pélissier P. Les interactions rural-urbain : circulation et mobilisation des ressources. $B$ APAD 2000 ; 19: 7-19.

Rogers EM. Diffusion of innovations $.5^{e}$ éd. New York: The Free Press, 2003.

Sanginga PC, Kamugisha RN, Martin AM. Conflicts management, social capital and adoption of agroforestry technologies : empirical findings from the highlands of southwestern Uganda. Agroforest Syst 2007; 69: 67-76.

Sanogo D. La haie vive dans le Sud bassin arachidier du Sénégal : adoption et conséquences agro-écologiques. Thèse, université Cheikh Anta Diop, Dakar, 2000.

Snelder DJ, Klein M, Schuren SHG. Farmers preferences, uncertainties and opportunities in fruit-tree cultivation in Northeast Luzon. Agroforest Syst $2007 ; 71: 1-17$ 